\title{
Implementación de actividades basadas en la Comprensión Lectora como estrategia para el fortalecimiento de la lectura crítica ${ }^{1}$
}

\section{Implementation of activities based on Reading Comprehension As a strategy for strengthening critical reading}

DOI: http://dx.doi.org/10.17981/cultedusoc.9.3.2018.34

Artículo de investigación. Fecha de recepción: 15/06/2018. Fecha de aceptación: 27/11/2018

\author{
Aurelio Larios Gómez; María Del Castillo; Lucia Jiménez Ortiz; \\ Elba Sinning Ríos; Ainne Rodríguez; José Terán Jaramillo; \\ Marina Martínez Caballero; Merlis Romero Ibarra; Donaldo Bastidas Yépez; \\ María Navarro Fontalvo; Alma Ramírez; Seyla Becerra Benítez; \\ Enna Fernández Vergara; Jorge Martínez España y Bertilda Rodríguez Ibarra² \\ Institución Educativa Distrital Institución Gilma Royero Solano (Colombia) \\ aulago777@hotmail.com
}

Para citar este artículo:

Larios, A., Del Castillo, M., Jiménez, L., Sinning, E., Rodríguez, A., Terán, J., Martínez, M., Romero, M., Bastidas, D., Navarro, M., Ramírez, A., Becerra, S., Fernández, E., Martínez, J. y Rodríguez, B. (2018). Implementación de actividades basadas en la Comprensión Lectora como estrategia para el fortalecimiento de la lectura crítica. Cultura. Educación y Sociedad 9(3), 303-308. DOI: http://dx.doi. org/10.17981/cultedusoc.9.3.2018.34

\section{Resumen}

La presente investigación aborda a la comprensión lectora como una de las competencias básicas que se deben adquirir a lo largo del periodo escolar, en este sentido, si los estudiantes no son capaces de comprender lo que leen, mucho menos podrán desarrollar una postura frente a lo mismo, por lo que es necesario desarrollar la comprensión lectora para contribuir a un entendimiento global de los textos. Para esto, se realizó un diagnóstico de los niveles de las competencias lectoras de los estudiantes de $3^{\circ}$ de la Institución Gilma Royero Solano, basado en resultados arrojados por las Pruebas Saber 2016, para luego de establecer una serie de estrategias enfocadas en la comprensión lectora, en el nivel de lectura crítica y aplicarlas al mismo grupo, con el fin de mejorar sus competencias lectoras y compararlas con los resultados obtenidos en pruebas realizadas por los docentes a partir de las Saber en el 2017.

Palabras clave: Comprensión lectora, lectura crítica, competencias lectoras.

\section{Abstract}

This research addresses reading comprehension as one of the basic skills that must be acquired throughout the school period, in this sense, if students are not able to understand what they read, much less can develop a position in front of what they read. same, so it is necessary to develop reading comprehension to contribute to a global understanding of the texts. For this, a diagnosis of the levels of reading skills of the students of 3rd of the Gilma Royero Solano Institution was made based on the results of the 2016 Saber Tests, after establishing a series of strategies focused on reading comprehension in the level of critical reading and apply them to the same group in order to improve their reading skills, compare them with the results obtained in tests made by teachers from the Saber in 2017.

Keywords: Reading comprenhension, critical reading, competences.

1 Este artículo ha sido derivado del Proyecto Fortalecimiento de la Cultura Ciudadana y Democrática en CT+I a través de la IEP apoyada en TIC en el Dpto. del Magdalena.

2 Docentes de la institución Educativa Distrital Institución Gilma Royero Solano

- The author; licensee Universidad de la Costa - CUC.

Cultura, Educación y Sociedad vol. 9 no. 3, pp. 303-308. Diciembre, 2018

Barranquilla. ISSN 2389-7724 Online 


\section{Introducción}

La comprensión lectora es una de las competencias básicas que se deben adquirir a lo largo del periodo escolar, sin embargo, es recurrente encontrar cómo se traduce en los niveles más bajos al revisar los resultados de las pruebas SABER de las instituciones. En este sentido, si los estudiantes no son capaces de comprender lo que leen, mucho menos podrán desarrollar una postura frente a lo mismo, por lo que es necesario desarrollar la comprensión lectora para contribuir a un entendimiento global de los textos.

Hoy en día hay una mayor preocupación por mejorar los niveles educativos en el país $\mathrm{y}$, sobre todo motivar a los establecimientos educativos a contribuir para mejorar los déficits de lectura que hoy se evidencian en los diferentes estudios; pero los resultados han arrojado que las campañas y propuestas planteadas no han contribuido tanto como se esperaba a mejorar los niveles de lectura comprensiva (Viñao, 2002).

Este es el caso particular de la Institución Educativa Técnica Departamental Gilma Royero Solano, en el que se presentan falencias respecto a esto a nivel analítico, inferencial, argumentativo e interpretativo, lo que se ha evidenciado tanto en los resultados de las pruebas SABER de $3^{\circ}$, como en el desarrollo de las clases.

Estos bajos niveles en esta competencia pueden ser el resultado de múltiples factores tales como el poco interés, la apatía, la desidia, la desmotivación, la falta de apoyo y acompañamiento de los padres de familia en los procesos formativos y la falta de control de los mismos, así como en el uso excesivo de tiempo que invierten los estudiantes en las redes sociales, de la mano con el mal manejo del tiempo libre.

Por este motivo, se considera necesario plantear y desarrollar estrategias para el desarrollo de la comprensión lectora; en tanto que, si el estudiante parte de comprender aquello que lee, podrá hacer una reflexión y lectura crítica sobre lo leído. De esta manera se podrán mejorar los resultados en las pruebas y el desempeño de los estudiantes en el aula.

\section{Comprensión lectora}

La comprensión lectora se enmarca dentro de las competencias comunicativas (ICFES, 2011), las cuales implican la capacidad de las personas de reconocer, comprender y dar una postura crítica sobre el contenido, la estructura e intencionalidad de la lectura (PISA, 2014). El desarrollo de este tipo de competencias conlleva a un mejor desempeño en cualquier área de conocimiento y contexto, ya sea educativo o laboral, entre otros (SEP, 2011).

La comprensión lectora va más allá de leer, es decir, de decodificar una serie de palabras y darles un significado global (Solé, 2010). Este es un proceso complejo $\mathrm{y}$ se ha desglosado en distintos niveles o secuencias de acciones, a partir de los cuales un texto puede ser comprendido teniendo en cuenta la habilidad del lector. Una comprensión lectora realizada a cabalidad, requiere hacer un acercamiento a lo que se lee, con el fin de poder realizar análisis, interpretaciones y reflexiones (Zorilla, 2005). Estos niveles son:

- Comprensión literal: Es el nivel más básico en el que el lector se limita a retener y recordar los aspectos relacionados con la lectura, tales como personajes, tiempo, lugar, ideas principales $y$ secundarias que se encuentran de manera explícita.

- Comprensión inferencial: Es un nivel más profundo de comprensión en el que se hace una lectura sobre los hechos más implícitos plasmados.

- Comprensión crítica: El lector es capaz de realizar una reflexión sobre lo leído, establecer su postura y dar argumentos para defenderla en función de sus conocimientos previos. 
El desarrollo de las comprensión lectora se refiere a "la capacidad de comprender, utilizar, reflexionar e interesarse por los textos escritos para alcanzar los propios objetivos, desarrollar el conocimiento y potencial personales, y participar en la sociedad" (OCDE, 2009: 14)

\section{Lectura Crítica}

Leer críticamente se puede entender como la aplicación de la lectura a la lógica analítica (Bernabeu, 2001). Leer es un proceso que implica distintos procesos como la visualización, la fonación, la audición y la comprensión que es permitida por el cerebro. En este sentido, de acuerdo a Dehaene (2015), los procesos que suceden en el cerebro permiten, finalmente, decodificar y dar significado a las palabras leídas como un todo, a nivel global, de manera asociada con factores sociales y culturales.

La lectura crítica es el nivel más profundo dentro de la comprensión lectora, e implica que el estudiante logre hacer un análisis y establezca una postura frente a lo leído, argumentando su posición. De acuerdo a Wallace (2009), la lectura crítica se rige por cuatro principios: en primer lugar, para la lectura de un texto se requiere de conocimientos previos, a nivel lingüístico y cultural, para poder realizar un análisis profundo; así mismo, el enfoque de lo leído varía en función del contexto; existen diferentes maneras de aproximarse a lo que se lee, así como diferentes propósitos; la lectura tiene un carácter social, en tanto permite la construcción de significados; y, finalmente, el fundamental es su carácter crítico.

Una adecuada lectura crítica implica lograr abstraer la intencionalidad del autor contenida en el texto de manera explícita e implícita, crear conocimiento y representaciones a partir de lo leído, no quedar ajeno al proceso (Serrano, 2008). Un lector crítico, de acuerdo a Ferreiro (2003), tiene la capacidad de identificar el tipo de texto que se encuentra leyendo, de interpretar el contenido, darle sentido en función de sus cogniciones, tomar decisiones y asumir una postura frente a esto.

\section{Metodología}

El artículo que se presenta es un producto derivado del estudio de la implementación de actividades basadas en la comprensión lectora como estrategia para el fortalecimiento de la lectura crítica. Se desarrolló en la Institución Gilma Royero Solano; su objetivo principal ha sido establecer una serie de estrategias enfocadas en la comprensión lectora en el nivel de lectura crítica.

El estudio contempla una metodología con un diseño de alcance propositivo. En primer lugar, se realizó un diagnóstico de los niveles de las competencias lectoras de los estudiantes de $3^{\circ}$ de la Institución Gilma Royero Solano, basado en los resultados arrojados por las Pruebas Saber 2016, para luego de establecer una serie de estrategias enfocadas en la comprensión lectora, en el nivel de lectura crítica y aplicarlas al mismo grupo y compararlas con los resultados obtenidos en evaluaciones realizadas por los docentes, con los mismos componentes de las pruebas Saber en 2017.

La investigación se desarrolló con un alcance propositivo, porque plantea y desarrolla estrategias para el desarrollo de la lectura crítica a partir de la comprensión lectora. Teniendo los distintos componentes evaluados en estas. Se evidencian avances progresivos en el proceso lector, comprensivo y crítico en los estudiantes en todos los niveles. En los niveles avanzados aumentaron el número de estudiantes, mientras que en el nivel insuficiente disminuyeron significativamente.

En su procedimiento se contó con la colaboración de estudiantes de $8^{\circ}$ grado. Dentro de las actividades realizadas para 
mejorar los niveles de lectura crítica partiendo de la comprensión lectora, se trabajó con cuentos, así como otros tipos de lecturas acordes a las edades y preferencias de los niños y se desarrollaron espacios para la reflexión colaborativa. De igual manera se tuvo participación activa de los padres en todo el proceso como público de las lecturas realizadas por sus hijos.

\section{Resultados}

Para comprobar los efectos benéficos de las estrategias empleadas, se presentan dos gráficos que permiten comparar los resultados entre los años 2016 y 2017, con el cual se mide el nivel de desempeño en la actividad lectora en sus distintos niveles.

En el año 2016, los estudiantes de $3^{\circ}$ de la institución obtuvieron los siguientes resultados:

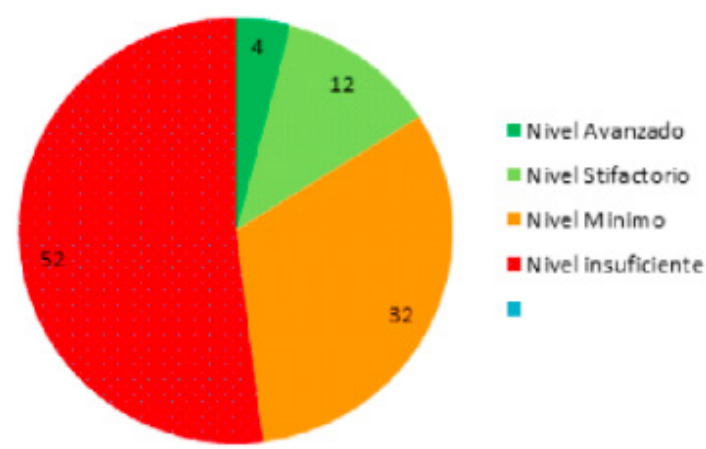

En el año 2017 , ya en $4^{\circ}$ y realizada la aplicación de las estrategias diseñadas, se obtuvieron los siguientes resultados:

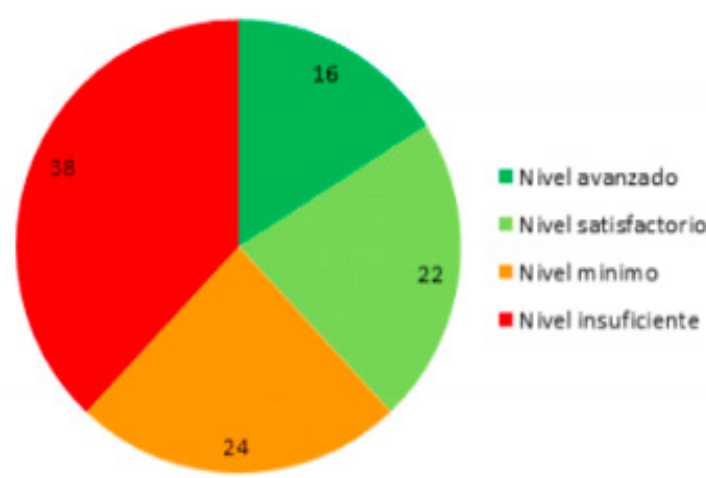

De esta manera se han evidenciado avances progresivos en el proceso lector, comprensivo y crítico en los estudiantes en todos los niveles. En los niveles avanzados aumentaron el número de estudiantes, mientras que en el nivel insuficiente disminuyeron significativamente.

\section{Conclusiones}

En general, los bajos niveles encontrados en competencia lectora pueden ser el resultado de múltiples factores, tales como el poco interés, la apatía, la desidia, la desmotivación, la falta de apoyo y acompañamiento de los padres de familia en los procesos formativos y la falta de control de los mismos, así como en el uso excesivo de tiempo que invierten los estudiantes en las redes sociales, de la mano con el mal manejo del tiempo libre.

En este aspecto se señala la evidencia en la falta de acompañamiento en ocasiones por partes de las instituciones; para lo cual Franco (2012) ve entre las causas que los docentes no utilizan estrategias que promuevan la comprensión lectora, porque invierten mucho tiempo en mantener a los estudiantes quietos, mientras que los docentes que realizan actividades sencillas y disponen del mayor número de estrategias para lograr niveles adecuados de comprensión; tienen mejores resultados.

Dentro de este estudio se manifiesta que no todos manejan las mismas herramientas para el fortalecimiento de la lectura y, para desarrollar estrategias en la institución, todos deben manejar estrategias, si se quiere potencializar la compresión lectora desde los primeros grados. Esto quiere decir que el desafío está en mejorar las competencias docentes en el manejo de las estrategias centradas en el aprendizaje del alumno.

En esta investigación para corroborar lo observado por los docentes, se tomó como evidencia las pruebas realizadas en el 
año 2016 a estudiantes de 3 grado de la institución, en cuyos resultados quedó demostrada la urgencia de intervenir en esta problemática.

A partir de esto se implementaron estrategias para mejorar los niveles de lectura crítica, que consistió en fortalecer la comprensión lectora por medio de cuentos, otros tipos de lecturas acordes a las edades y preferencias de los niños, y desarrollar espacios para la reflexión colaborativa sobre estos. De igual manera se contó con la participación activa de los padres en todo el proceso como público de las lecturas realizadas por sus hijos.

Posteriormente, luego de realizar las estrategias anteriormente planteadas, los mismos estudiantes presentaron, en 2017, la prueba correspondiente y los resultados obtenidos, dieron cuenta del éxito de las estrategias utilizadas debido a que de 52 estudiantes evaluados en 2016, se encontraron en un nivel insuficiente, mientras que en el siguiente año solo 38 estudiantes quedaron en este nivel.

Las estrategias deben ser realizadas, con base en las oportunidades y falencias que se presentan directamente en el aula. A continuación, se presentan algunas recomendaciones:

En primer lugar, solo se enfocan en el propósito inicial de esta etapa de la lectura y esto se lleva a cabo con el reconocimiento de grafemas y sus combinaciones, se basan en la relación grafema-morfema. En otras palabras, no utilizan estrategias más complejas y estructuradas para suscitar en los niños otro tipo de ejercicios mentales que favorezcan la comprensión de los textos. Además de ello, se puede advertir que la práctica que predomina por parte de los docentes orienta la manera de asumir la actividad lectora, por parte del alumno, a tal punto que los estudiantes no disfrutan el propósito de la actividad (Franco, 2012).
En segunda instancia, se establece el concepto del desarrollo de pensamiento crítico porque es una de las habilidades fundamentales que deben cultivar los estudiantes de este milenio. En relación con esto, se resalta que la criticidad es una actitud frente a la vida y al mundo y a la vez una manera de pensar, y que la lectura como experiencia formativa facilita construcciones culturales desde la escuela (García y Arrieta, 2016).

El pensamiento crítico es fundamental en el desarrollo de la lectura y es base en la formación de estrategias que beneficien a la misma. El sistema educativo pretende fomentar el desarrollo de la competencia lectora de manera comprensiva, y para ello parte de la afirmación de Ausubel (1980), según la cual el "lenguaje escrito" es la "llave" no solo del ingreso a un mundo alfabetizado, sino la oportunidad de aprender de manera significativa desde la lectura y así comprender su realidad para transformarla (Franco, 2012).

En ese mismo orden, para que las estrategias puedan ser establecidas y ejecutadas de la mejor manera se tiene en cuenta la siguiente afirmación sobre la lectura crítica, en donde es tarea urgente del docente propiciar intencionalmente la lectura crítica, no como un proceso de imposición de lecturas, sino como una actividad de desarrollo cognitivo, comprensivo, argumentativo, participativo y emancipatorio, que garantice herramientas de disertación, metacomprensión para encuentros socio-culturales desde el texto hacía la sociedad y viceversa.

Todo parte que la lectura en general es vista como una necesidad imperiosa del mundo de hoy. Lo importante es que sea considerada como una de las habilidades lingüísticas que presenta más falencia a nivel de educación básica y media en el país. Así lo demuestra el bajo índice de desarrollo de pensamiento crítico de los estudiantes en las pruebas internacionales y nacionales (García y Arrieta, 2016). 
Asimismo, Avendaño y Martínez (2013) señalan la importancia que tienen las TIC para la enseñanza y aprendizaje de la lectura, las autoras consideran que la generación de nuevos escenarios permite al estudiante apropiarse de las competencias lectoras.

En conclusión y teniendo en cuenta los aportes de cada autor, con respeto a las falencias y lo que se tiene que tener en cuenta para la elaboración y ejecución pertinente de las estrategias de la compresión lectora, se debe mantener el adecuado acompañamiento y seguimiento de la misma, para que el cumplimiento y la implementación de la investigación sea de gran avance en el desarrollo de la compresión lectora.

\section{Referencias}

Ausubel, D. (1980). Psicología educativa: un punto de vista cognoscitivo. ( $4^{\mathrm{a}}$ Edición). México, D.F.: Ed. Trillas.

Avendaño, I. y Martínez, D. (2013). Competencia lectora y el uso de las nuevas tecnologías delainformaciónycomunicación. Revista Escenarios, 11(1), 7-22.

Bernabeu, N. (2001). Una propuesta creativa de animación. Cuadernos de literatura infantil y juvenil, 14(136). 26-35

Dehaene S. et al. (2015). Illiterate to literate: behavioral and cerebral changes induced by reading acquisition. Nature Review Neuroscience, 16(4). 234-244.

Franco, M. (2012). Estrategias de enseñanza para la promoción de la comprensión lectora desde el aprendizaje significativo. Cultura, Educación y Sociedad 3(1), 175-18

Ferreiro, E. (2003). La escuela no forma buenos lectores. Diario La Nación.
García, H. y Arrieta, A. (2016). Potencialización del pensamiento crítico a través de la hipotemetacomprensión textual (hmt). Cultura Educación y Sociedad ,7(2), 54-71.

Molina, S. (1984). Batería diagnóstica de la Madurez para la Lectura. Madrid, CEPE .

Organización para la Cooperación y el Desarrollo Económicos-OCDE. (2009). Pisa 2009 Assessment Framework Key Competencies in Reading, Mathematics and Science. París: OCDE

PISA. (2014). Programa Internacional de Evaluación de Estudiantes. (Tercera ed.) Santiago de Chile: Agencia de Calidad de la Educación.

SEP. (2011). Manual de procedimiento para el fomento y la valoración de la competencia lectora en el Aula. México, D.F.: Secretaría de Educación Pública

Serrano, S. (2008). El desarrollo de la comprensión crítica en los estudiantes universitarios: hacia una propuesta didáctica. Mérida: Universidad de los Andes.

Solé, I. (2012). Competencia lectora y aprendizaje. Revista Iberoamericana de Educación (OEI), 59. 43-61.

Viñao, A. (2002) La enseñanza de la lectura y la escritura: análisis socio-histórico. Anales de documentación, 5(1), 345-359.

Wallace, C. (2009). EAL Learners and Critical Reading. Reader in Education, Institute of Education, 12-17. University of London, UK 